С. Сабер${ }^{1}$, M. Houshmand ${ }^{2}$, M. Eftekharzadeh ${ }^{3}$, M.R. Samiei Nasab ${ }^{4}$, A.F. Fazelifar ${ }^{5}$, M. Haghjoo ${ }^{5}$, Е.В. Заклязьминская ${ }^{1,6}$, А.В. Гавриленко ${ }^{1,6}$

\footnotetext{
${ }^{1}$ Первый Московский государственный медицинский университет им. И.М. Сеченова, Российская Федерация ${ }^{2}$ Национальный институт генной инженерии и биотехнологии, Тегеран, Иран

${ }^{3}$ Клиника Аритмии, Тегеран, Иран

Тегеранская клиника аритмий, Иран

${ }^{4}$ Chamran Heart Hospital, Университет медицинских наук, Исфахан, Иран

${ }^{5}$ Shaheed Rajaei Cardiovascular, Medical and Research Center, Tehran, Iran

${ }^{6}$ Российский научный центр хирургии им. акад Б.В. Петровского, Москва, Российская Федерация

\section{Клинический полиморфизм и подходы к лечению нарушений ритма сердца в семье с делецией $\triangle \mathrm{KPQ} 1505-1507$ в гене $S C N 5 A$}

Цель исследования: изучить спектр клинических проявлений и ответов на лечение в большой семье с нарушениями ритма, обусловленными p.delKPQ1505-1507 в гене SCN5A. Пациенты и методы: были обследованы 18 членов большой иранской семьи, имеющие различные комбинации наследственных нарушений ритма сердиа. Членам семьи было проведено полное кардиологическое обследование, медико-генетическое консультирование, а также были получены образцы венозной крови для выполнения молекулярно-генетического исследования. Поиск мутаций в гене SCN5A был осуществлен методом прямого секвенирования по Сенгеру. Результаты: в иранской семье была выявлена известная мутация (p.delKPQ 1505-1507) в гене SCN5A. Интересно, что среди членов семьи-носителей этой мутации присутствовали больные как c LQ-TS, так и с синдромом Бругада. Выводы: перекрывание фенотипов, связанных с высоким риском внезапной сердечной смерти, может потребовать комплексного подхода к антиаритмической терапии, хирургическому лечению и профилактике внезапной сердечной смерти в этой семье.

Ключевые слова: синдром удлиненного интервала $Q-T$, синдром Бругада, SCN5A, имплантация кардиовертера-дефибриллятора, антиаритмическая терапия.

(Вестник РАMH. 2014; 5-6: 52-59)

S. Saber' ${ }^{1}$, M. Houshmand ${ }^{2}$, M. Eftekharzadeh ${ }^{3}$, M.R. Samiei Nasab ${ }^{4}$, A.F. Fazelifar ${ }^{5}$, M. Haghjoo ${ }^{5}$, E.V. Zaklyazminskaya ${ }^{1,6}$, A.V. Gavrilenko ${ }^{1,6}$

${ }^{1}$ Sechenov First Moscow State Medical University, Moscow, Russian Federation

${ }^{2}$ National Institute of Genetic Engineering and Biotechnology, Tehran, Iran

${ }^{3}$ Tehran Arrhythmia Clinic, Iran

${ }^{4}$ Chamran Heart Hospital, Isfahan University of Medical Sciences, Iran

${ }^{5}$ Shaheed Rajaei Cardiovascular, Medical and Research Center, Tehran, Iran

${ }^{6}$ Petrovsky Russian Research Centre of Surgery, Moscow, Russian Federation

\title{
Clinical Polymorphisms and Approaches of Arrhythmias Treatment in a Family with $\triangle$ KPQ1505-1507 Deletion in SCN5A Gene
}

Background: The aim of the study was to analyze spectrum of manifestation and treatment response in large family with rhythm disturbances caused by p.delKPQ1505-1507 mutation in SCN5A gene. Patients and methods: We had under our observation 18 members of large Iranian family with various combination of inherited arrhythmic syndromes. Careful cardiological examination, genetic councelling and venous blood sampling for molecular genetic study were performed for family members. Mutation screening in SCN5A gene was performed using bidirectional Sanger sequencing. Results: Here by we show the observation of Iranian family with known mutation p.delKPQ 1505-1507 in SCN5A gene, who display not only $L Q-T S$ phenotype but also some of the carriers of this mutation have had LQ-TS and Brugada syndrome (combine phenotype), interestingly. Conclusion: The overlapping phenotype associated with high risk of sudden cardiac death may require complex approaches to antiarrhythmic therapy, surgical treatment and prevention of sudden cardiac death in the family.

Key words: Long Q-T syndrome, Brugada syndrome, SCN5A, implantable cardioverter-defibrillator, antiarrhythmic therapy. (Vestnik Rossiiskoi Akademii Meditsinskikh Nauk - Annals of the Russian Academy of Medical Sciences. 2014; 5-6: 52-59) 


\section{Введение}

Заболевания сердечно сосудистой системы являются одной из наиболее распространенных причин смерти в развитых странах. К сожалению, точной статистической информации о распространенности внезапной сердечной смерти (ВСС) в России и Иране нет.

Около $20 \%$ всех случаев ВСС приходится на нарушения ритма сердца, которые при отсутствии структурных изменений в миокарде классифицируют как идиопатические. К ним относят синдромы удлиненного интервала $Q-T$, короткого интервала $Q-T$, синдром Бругада (СБ), Леви-Ленегра, синдром детской внезапной смерти, идиопатическую желудочковую тахикардию [1-4].

За последние два десятилетия накоплены данные о генетической природе этих заболеваний. Поскольку большинство из них обусловлены мутациями в генах ионных каналов, их объединили в группу т.н. каналопатий $[5,6]$. Наиболее частым и хорошо изученным заболеванием в этой группе является синдром удлиненного интервала $Q-T(L Q-T S)$. Частота первичной формы синдрома удлиненного интервала $Q-T$ составляет 1:2500-1:5000 [7]. Диагноз $L Q-T S$ ставят при увеличении продолжительности интервала $Q-T$ на ЭКГ свыше максимального значения для данной частоты сердечных сокращений на $10 \%$. В качестве абсолютного диагностического критерия используют корригированный интервал $Q-T(Q-T c)$, рассчитываемый по формуле Базетта [6, 8]. Превышение $Q-T c>470$ мс у мужчин и $>480$ мс у женщин является диагностически значимым. При этом заболевании могут развиваться пароксизмы желудочковой тахикардии по типу «пируэта» (torsade de pointes), в ряде случаев переходящие в фибрилляцию желудочков $[9,10]$.

СБ был описан относительно недавно, в 1992 г. [11]. Заболевание характеризуется блокадой правой ножки пучка Гиса, подъемом сегмента $S T \geq 2$ мм в отведениях $\mathrm{V}_{1}-\mathrm{V}_{2}$, синкопальными состояниями и высоким риском ВСС вследствие развития приступов полиморфной желудочковой тахикардии [2, 12].

Частота СБ в Российской Федерации точно не определена, однако было проведено большое скрининговое исследование в Самарской обл., согласно результатам которого встречаемость Бругада-паттерна на ЭКГ составляет $\approx 1: 10000$ [13].

Эти данные в целом совпадают с оценками частоты встречаемости СБ в Европе и США [7]. В Иране строгих эпидемиологических исследований не проводилось. В настоящее время известно по крайней мере 12 генов, ответственных за $L Q-T S$; за развитие СБ, по последним оценкам, отвечает 17 генов (табл. 1) [14, 15]. Мутации в трех генах - CACNAIC, KCNH2 и SCN5A- могут приводить к обоим заболеваниям.

При СБ мутации в гене $S C N 5 A$ обнаруживают у $15-30 \%$ больных, тогда как вклад $S C N 5 A$-опосредованных случаев в структуру $L Q-T S$ составляет $5-10 \%[7,16]$. В гене $S C N 5 A$ описано более 500 мутаций, из них около 100 ответственны за $L Q-T S$ и более 370 связаны с СБ $[12,17]$.

Многочисленные электрофизиологические исследования на модельных клетках показали, что мутации, идентифицированные у больных с $L Q-T S$, в основном приводят к замедленной инактивации $\mathrm{Na}^{+}$-канала либо

Таблица 1. Гены, ответственные за синдром удлиненного интервала $Q-T$ и синдром Бругада

\begin{tabular}{|c|c|c|c|}
\hline Структурные субъединицы & Ген & Заболевание & Частота выявления мутаций, \% \\
\hline \multirow{11}{*}{$\begin{array}{l}\text { Структурные субъединицы } \\
\text { и регуляторные белки } \mathrm{Na}+- \\
\text { каналов }\end{array}$} & $S C N 5 A$ & $\begin{array}{c}\text { CБ } 1 \\
\text { LQ-TS } 3\end{array}$ & $\begin{array}{c}11-28 \\
5\end{array}$ \\
\hline & GPD1-L & СБ 2 & $<1$ \\
\hline & $S C N 1 B$ & СБ 5 & $<1$ \\
\hline & $S C N 3 B$ & СБ 7 & $<1$ \\
\hline & MOG1 & СБ 11 & $<1$ \\
\hline & SLMAP & СБ 15 & $<1$ \\
\hline & $S C N 2 B$ & СБ 17 & $<1$ \\
\hline & CAV3 & LQ-TS 9 & $<1$ \\
\hline & $S C N 4 B$ & LQ-TS 10 & $<1$ \\
\hline & SNTA1 & LQ-TS 12 & $<1$ \\
\hline & $A N K B$ & LQ-TS 4 & $<1$ \\
\hline \multirow{11}{*}{$\begin{array}{l}\text { Структурные субъединицы К+- } \\
\text { каналов }\end{array}$} & $K C N Q 1$ & LQ-TS 1 & 40 \\
\hline & KCNH2 & $\begin{array}{c}\text { CБ } 8 \\
\text { LQ-TS } 2\end{array}$ & $\begin{array}{l}<1 \\
15\end{array}$ \\
\hline & KCNE1 & LQ-TS 5 & $<1$ \\
\hline & KCNE2 & LQ-TS 6 & $<1$ \\
\hline & KCNE3 & СБ 6 & $<1$ \\
\hline & KCNE5 & СБ 12 & $<1$ \\
\hline & KCNJ2 & LQ-TS 7 & $<1$ \\
\hline & KCNJ5 & LQ-TS 13 & $<1$ \\
\hline & KCNJ8 & СБ 9 & $<1$ \\
\hline & KCND3 & СБ 13 & $<1$ \\
\hline & HCN4 & СБ 14 & $<1$ \\
\hline \multirow[t]{4}{*}{$\begin{array}{l}\text { Структурные субъединицы } \\
\text { Са+-каналов }\end{array}$} & $C A C N A 1 c$ & $\begin{array}{c}\text { CБ } 3 \\
\text { LQ-TS } 8\end{array}$ & $\begin{array}{c}3-4 \\
<1\end{array}$ \\
\hline & $C A C N B 2 b$ & СБ 4 & $2-3$ \\
\hline & CACNA2D1 & СБ 10 & $<1$ \\
\hline & TRMP4 & СБ 16 & $<6$ \\
\hline
\end{tabular}


I

II

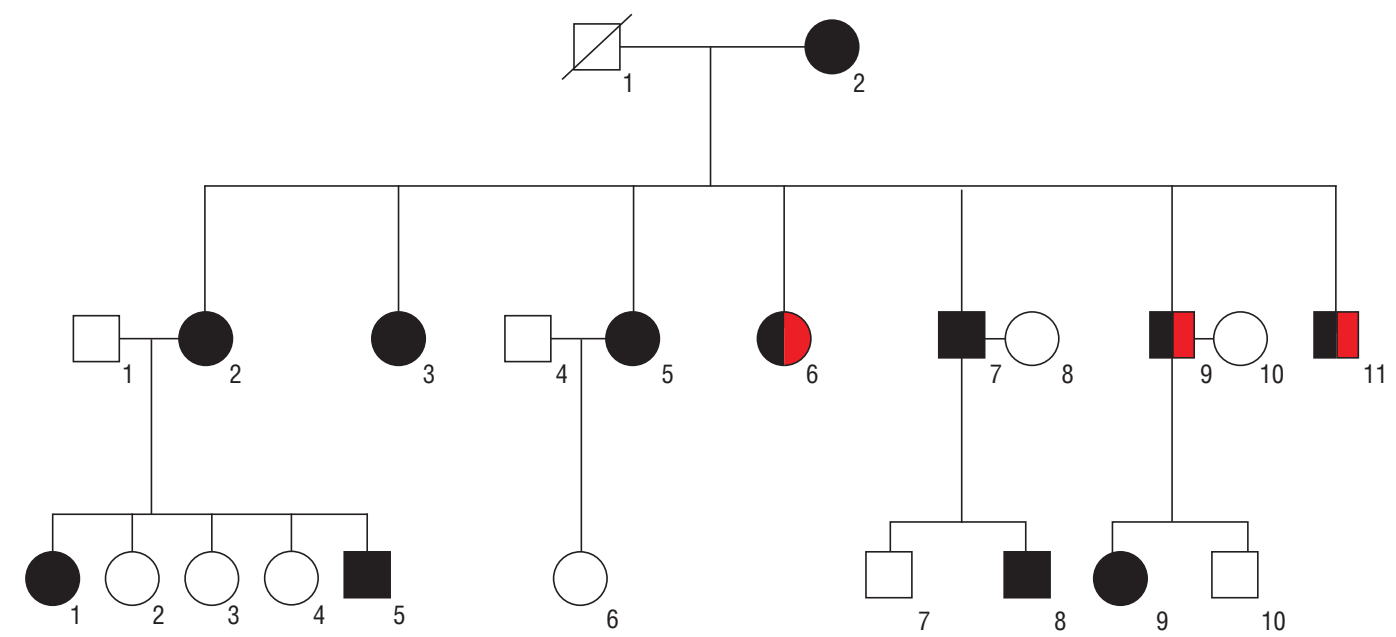

LQ-TS

Синдром Бругада и LQ-TS

Рис. 1. Родословная обследованной семьи.

Примечание. Римскими цифрами обозначены номера поколений, арабскими - порядковый номер человека в поколении. Пробанд указан стрелкой.

к появлению устойчивого позднего $\mathrm{Na}^{+}$-тока во время плато-фазы потенциала действия сердца [18]. Мутации гена $S C N 5 A$ обсуловливают широкий спектр клинических фенотипов, включающих, помимо СБ и $L Q-T S$, болезнь Леви-Ленегра (прогрессирующее нарушение проводимости), идиопатическую фибрилляцию желудочков, синдром детской внезапной смерти, врожденный синдром слабости синусового узла, семейную форму фибрилляции предсердий, аритмогенную кардиомиопатию правого желудочка и дилатационную кардиомиопатию [19, 20].

Патофизиологическим механизмом развития СБ при мутациях в гене SCN5A является уменьшение числа или ускоренная инактивация натриевых каналов в клетках эпикарда правого желудочка, приводящие к снижению плотности потока натрия и преждевременной реполяризации эпикарда [2, 12]. Терапия этих двух заболеваний также различна. Общепринятым лечением при $L Q-T S$ является назначение $\beta$-блокаторов [21]. Однако эти препараты имеют ограниченную эффективность при лечении $L Q-T S$, поскольку не менее $32 \%$ пациентов с этой формой заболевания продолжают иметь синкопе и жизнеугрожающие желудочковые события, несмотря на прием $\beta$-блокаторов [22]. Именно поэтому при симптомном течении синдрома удлиненного интервала $Q-T$, вызванного мутациями в гене $S C N 5 A(L Q-T S)$, имплантация кардиовертера-дефибриллятора (ИКД) рассматривается рядом аритмологов в качестве первой линии терапии [23], более эффективной, чем назначение $\beta$-блокаторов [24].

При лечении СБ ни один из представленных в настоящее время на рынке антиаритмических препаратов не был признан надежно снижающим смертность от желудочковых аритмий [11]. Имеются изолированные публикации о снижении выраженности синдрома ранней реполяризации желудочков и предотвращении развития аритмий при назначении хинидина, изопротеренола, а также при использовании учащающей электростимуляции желудочков как в клинической практике, так и в исследованиях
$[25,26]$. Однако единственным достоверно эффективным методом профилактики ВСС у симптомных больных с СБ в настоящее время остается ИКД [27, 28]. Существуют изолированные свидетельства того, что назначение амиодарона при ИКД может уменьшить частоту срабатываний, хотя эффективность амиодарона для предотвращения аритмий, как было уже сказано выше, не доказана.

Два аллельных заболевания, $L Q-T S$ и СБ типа 1, являются результатом функционально противоположных нарушений работы натриевого канала. Однако интересно, что в гене $S C N 5 A$ известны уникальные генетические варианты (p.1795insD, p.E1784K и p.delKPQ 1505-1507), приводящие к развитию обоих заболеваний, причем даже среди членов одной семьи [29-31].

Цель исследования: изучить спектр клинических проявлений и ответов на лечение в большой семье с нарушениями ритма сердца, обусловленными p.delKPQ1505-1507 в гене $S C N 5 A$.

\section{Пациенты и методы}

\section{Участники исследования}

Под нашим наблюдением находилась иранская семья, в которой наблюдались нарушения сердечного ритма и случаи ВСС. Для кардиологического и генетического исследования были доступны 18 человек (рис. 1).

Исследование проведено в соответствии с протоколом, утвержденным Этическим комитетом РНЦХ им. акад. Б.В. Петровского. От каждого члена семьи было получено письменное информированное согласие на участие в исследовании. Обследование включало обзор истории болезни, полный медицинский осмотр, разовую двенадцатиканальную ЭКГ покоя, двадцатичетырехчасовое холтеровское мониторирование ЭКГ и УЗИ сердца.

\section{Методы исследования}

Диагнозы $L Q-T S$ и СБ были поставлены на основании общепринятых диагностических критериев [2, 9]. 
Все члены семьи отказались от проведения нагрузочной фармакологической пробы с прокаинамидом или флекаинидом (антиаритмические классы Іа и Ic). Генетическое обследование включало сбор и анализ родословной, взятие венозной крови для проведения ДНК-диагностики. Образцы ДНК были выделены из венозной крови стандартным методом фенол-хлороформной экстракции. Поиск мутаций был выполнен в кодирующих экзонах гена $S C N 5 A$ и прилегающих интронных областях. Последовательность олигопраймеров может быть предоставлена по запросу. Поиск генетических вариантов производили методом прямого секвенирования по Сенгеру с использованием анализатора ABI300 (Life Technology, США). Частоту выявленных генетических вариантов оценивали методом ПДРФ-анализа или аллельспецической ПЦР в группе здоровых добровольцев (100 человек; 200 хромосом) соответствующей этнической принадлежности.

\section{Результаты}

Пробанд (первый обратившийся за консультацией член семьи) - мужчина, 54 года, перенесший эпизод ВСС в возрасте 50 лет с успешной реанимацией. На ЭКГ были выявлены удлинение интервала $Q-T c$ до 533 мс, удлинение интервала $P R$ до 220 мс, спонтанная элевация сегмента $S T \geq 2$ мм в отведениях $\mathrm{V}_{1}-\mathrm{V}_{3}$. При тщательном клиническом обследовании 18 членов семьи диагноз наследственных нарушений ритма был поставлен 12 больным. Примечательно, что у пробанда и двоих его родственников (II.11 и II.6) были одновременно выявлены признаки $L Q-T S$ и СБ (рис. $1-3$ ). У 9 членов семьи (I.2, II.2, II.3, II.5, II.7, III.1, III.5, III.8, III.9) определили только диагностически значимое удлинение интервала $Q-T$ (см. рис. 1). Соотношение больных по полу составило 5:7 (М:Ж). Средняя продолжительность ин- тервала $Q T c$ оказалась выше у членов семьи с комбинацией симптомов СБ и $L Q-T S(510 \pm 32,3$ мс) в отличие от тех, которые имели только удлинение интервала $Q-T$ $(490 \pm 13,9$ мс). У всех больных в этой семье зубец $T$ был значительно удален от $Q R S$-комплекса, и наблюдалась его альтернация.

Основными жалобами больных были сердцебиения и головокружения при эмоциональной нагрузке, шуме и после еды. Больным II.7, II.11 и III.8 было проведено УЗИ сердца; структурных изменений обнаружено не было. Базовой терапией при синдроме удаленного интервала $Q-T$ является назначение $\beta$-блокаторов. Пятеро членов семьи (II.2, II.9, II.11, III.1, III.8) регулярно принимали пропранолол. На фоне приема пропранолола в дозе 40 мг/сут частота и выраженность симптомов (головокружение, сердцебиение) уменьшились у всех наблюдаемых. Нарастания клинической симптоматики с возрастом отмечено не было.

В результате поиска мутаций в гене $S C N 5 A$ была идентифицирована делеция p.delKPQ1505-1507 у пробанда и 11 членов семьи, имеющих симптомы СБ и/или $L Q-T S$. Ни один из клинически здоровых родственников не был носителем мутации.

Всем членам семьи провели медико-генетическое консультирование. Пациентам были объяснены особенности аутосомно-доминантного типа наследования СБ и $L Q-T S, 50 \%$ риск передачи заболевания потомкам. Также они были информированы о наличии средовых факторов риска, характерных для $L Q-T S$ (прием ряда лекарственных препаратов) и СБ (гипертермия, алкоголь).

Всем носителем мутации p.delKPQ1505-1507 рекомендовали имплантацию кардиовертера-дефибриллятора, однако к настоящему моменту хирургическое лечение проведено только 2 членам семьи (II.2 и II.9), имевшим в анамнезе эпизоды ВСС с успешной реанимацией (табл. 2).
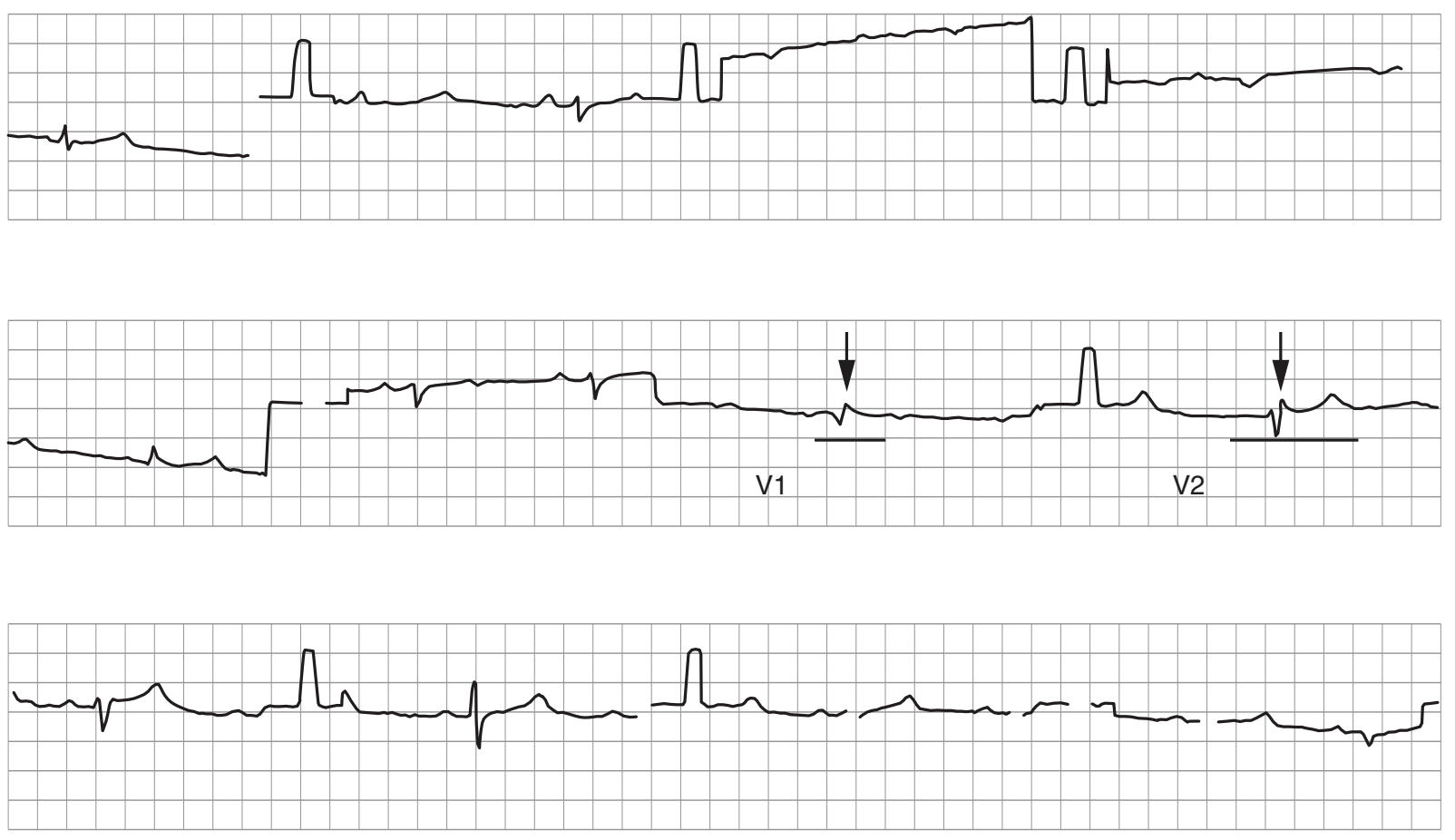

Рис. 2. Фрагмент ЭКГ пробанда (02-II-9).

Примечание. Характерный Бругада-паттерн указан стрелками $\left(\mathrm{V}_{1}-\mathrm{V}_{2}\right)$, ЧСС 58 уд./мин, $Q-T c 504$ мс, PR 220 мс. 

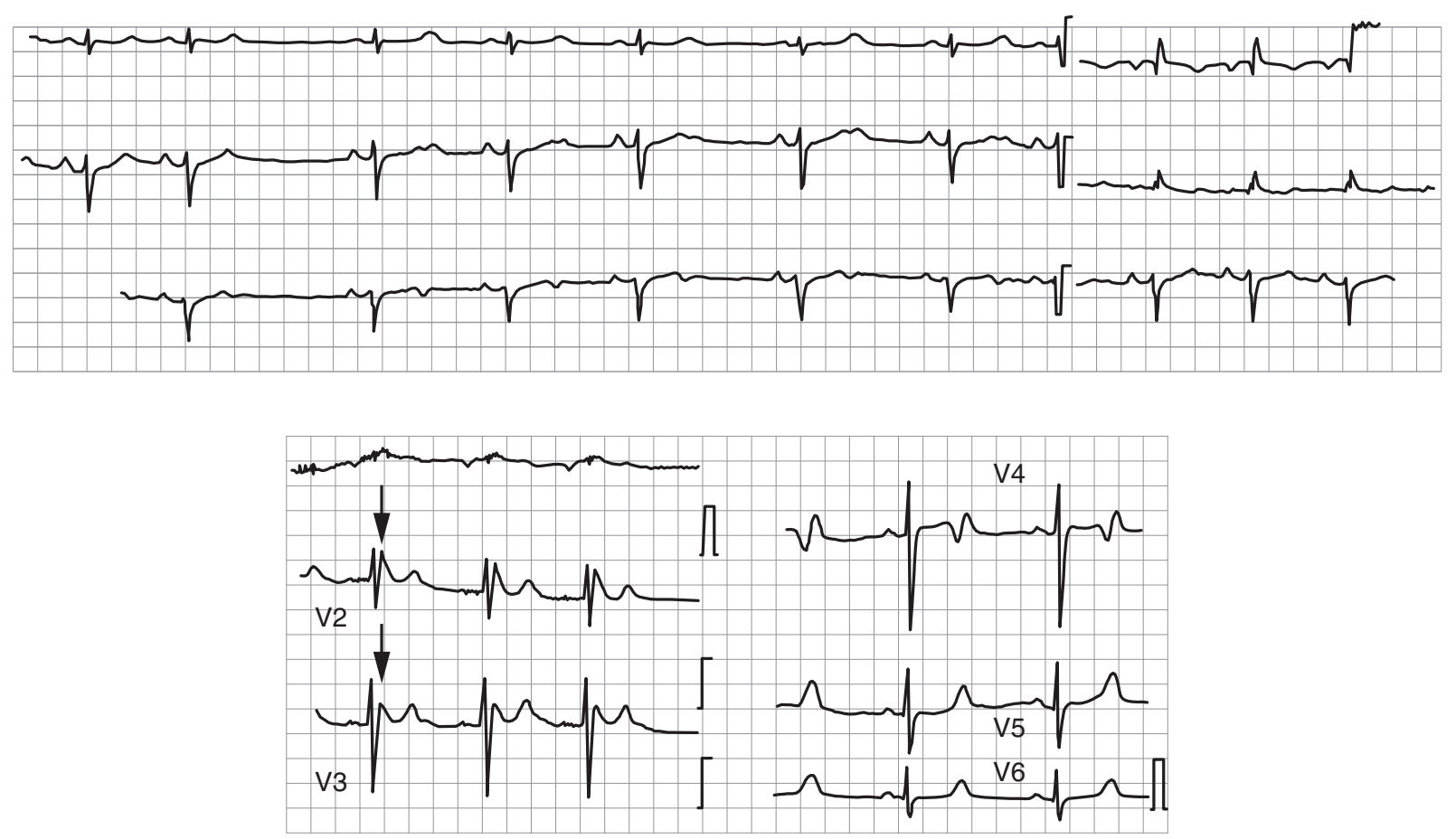

Рис. 3. Фрагмент ЭКГ пробанда (02-II-11).

Примечание. Характерный Бругада-паттерн указан стрелкой $\left(\mathrm{V}_{2}-\mathrm{V}_{3}\right)$.ЧСС 62 уд./мин, $Q-T c 524$ мс, PR 200 мс.

Таблица 2. Клинические особенности членов больных с выявленной мутацией p.delKPQ 1505-1507 в гене SCN5A

\begin{tabular}{|c|c|c|c|c|c|}
\hline Шифр & Пол & Диагноз & Q-Tc, мс & PR, мс & Лечение \\
\hline $\mathrm{I}-2$ & ж & LQ-TS & 500 & 220 & Рекомендована ИКД \\
\hline II -2 & Ж & LQ-TS & 485 & 180 & $\begin{array}{l}\text { ИКД + } \beta \text {-блокатор (пропранолол } 40 \text { мг/сут - поддерживающая доза, и до- } \\
\text { полнительно разовый прием } 40 \text { мг при сердцебиениях) } \\
\text { Кардиовертер-дефибриллятор был имплантирован в декабре } 2011 \text { г. } \\
\text { В последующем: каждые } 6 \text { мес } \\
3 \text { неожиданных / спонтанных шока в течение первого года }\end{array}$ \\
\hline II-3 & Ж & LQ-TS & 480 & 200 & Рекомендована ИКД \\
\hline II -5 & ж & LQ-TS & 520 & 180 & Рекомендована ИКД \\
\hline II -6 & Ж & LQ-TS + СБ & 473 & 200 & Рекомендована ИКД \\
\hline II-7 & M & LQ-TS & 480 & 190 & Рекомендована ИКД \\
\hline $\begin{array}{c}\text { II-9 } \\
\text { Пробанд }\end{array}$ & M & LQ-TS + СБ & 533 & 220 & $\begin{array}{l}\text { ИКД + } \beta \text {-блокатор (пропранолол } 40 \text { мг/сут - поддерживающая доза и допол- } \\
\text { нительно разовый прием } 40 \text { мг при сердцебиениях) } \\
\text { Кардиовертер-дефибриллятор был имплантирован в } 2008 \text { г. } \\
\text { В последующем: каждые } 6 \text { мес } \\
5 \text { немотивированных и } 1 \text { мотивированный шок (в } 2011 \text { и по настоящее время) }\end{array}$ \\
\hline II-11 & M & LQ-TS + СБ & 524 & 200 & $\begin{array}{l}\text { Рекомендована ИКД + } \beta \text {-блокатор (пропранолол } 40 \text { мг/сут - поддерживаю- } \\
\text { щая доза и дополнительно разовый прием } 40 \text { мг при сердцебиениях) }\end{array}$ \\
\hline III-1 & ж & LQ-TS & 480 & 180 & $\begin{array}{l}\text { Рекомендована ИКД + } \beta \text {-блокатор (пропранолол } 40 \text { мг/сут - поддерживаю- } \\
\text { щая доза и дополнительно разовый прием } 40 \text { мг при сердцебиениях) }\end{array}$ \\
\hline III-5 & M & LQ-TS & 480 & 180 & Рекомендована ИКД \\
\hline III-8 & M & LQ-TS & 500 & 200 & $\begin{array}{l}\text { Рекомендована ИКД + } \beta \text {-блокатор (пропранолол } 40 \text { мг/сут - поддерживаю- } \\
\text { щая доза и дополнительно разовый прием } 40 \text { мг при сердцебиениях) }\end{array}$ \\
\hline III-9 & Ж & LQ-TS & 485 & 160 & Рекомендована ИКД \\
\hline
\end{tabular}

\section{Обсуждение}

Электрофизиологические свойства делеции p.delKPQ1505-1507 и ее роль в аритмогенезе были впервые изучены группой Nuyens и соавт. в 2001 г. на экспериментальной модели мышей, гетерозиготных по мутации p.delKPQ1505-1507 [32, 33]. Делеция расположена в непо- средственной близости от кластера из трех аминокислот (Ile-Phe-Met, IFM), которые входят в активный центр быстрой инактивации канала [34]. В результате делеции происходит нарушение быстрой инактивации, увеличение входящего натриевого тока INa, большей деполяризации во время потенциала действия, что и проявляется феноменом удлинения интервала $Q-T$ на ЭКГ [35]. Именно 
поэтому наличие у носителей делеции синдрома удлиненного интервала $Q-T$ является предсказуемым. Однако присоединение Бругада-феномена на ЭКГ у двух членов семьи нуждается в дополнительном объяснении.

На наш взгляд, наиболее вероятно модифицирующее влияние дополнительного генетического фактора (второй мутации в другом гене, ответственном за СБ). Второй генетический вариант, ответственный за СБ, мог быть унаследован от члена семьи (I.1). К сожалению, клинические данные и биологический материал этого родственника не были нам доступны, т.к. он умер до начала обследования семьи. Косвенным свидетельством в пользу этой гипотезы является тот факт, что оба носителя комбинированного фенотипа являются потомками одних родителей и могли унаследовать одинаковую комбинацию мутаций. Дочь (III.9) пациента (II.9) унаследовала только один признак, удлинение интервала $Q-T$, что также не противоречит нашей гипотезе: у нее было подтверждено носительство делеции, ответственной за $L Q-T S$, а дополнительный генетический вариант она не унаследовала.

Наличие компаунд-гетерозигот не является необычным для $L Q-T S$. По данным американского регистра ИКД, их доля составляет от 4 до $8 \%$ среди пациентов с имплантированными антиаритмическими устройствами [14]. Имеются единичные публикации о носительстве двух мутаций при СБ, но данные о частоте гетерозиготных компаундов при этом заболевании пока отсутствуют [36].

Как известно, при $L Q-T S$ и СБ факторы риска развития желудочковой тахикардии различны. Повышение температуры при СБ, как эндогенное, так и средовое (пребывание в жарком климате, в бане и т.д.), является фактором риска развития желудочковой тахиаритмии и ВСС, но не оказывает большого влияния на $L Q-T S$. Больные с удлинением интервала $Q-T$ чувствительны к физическим и эмоциональным нагрузкам (особенно плаванию), резким звукам. Списки лекарственных препаратов, не показанных к применению в течение всей жизни при этих заболеваниях, также различаются $[37,38]$.

Мы считаем, что больных, у которых имеет место комбинация симптомов обоих заболеваний, также необходимо информировать об избежании максимально полного спектра факторов риска, характерных для обоих заболеваний. Хотя эффективность $\beta$-блокаторов при $L Q-T S$ ограничена, а при СБ их эффективность не подтверждена, у членов семьи с делецией p.delKPQ1505-1507 мы наблюдали значимое уменьшение выраженности клинических симптомов при приеме пропранолола. ИКД была рекомендована пациентам с целью профилактики ВСС, в особенности больным с комбинированным фенотипом.

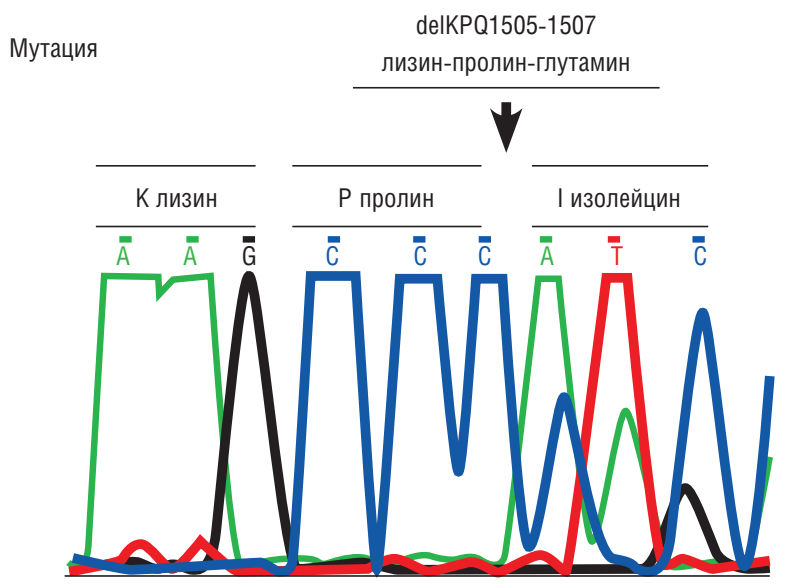

Рис. 4. Фрагмент прямого секвенирования по Сенгеру экзона 26 гена $S C N 5 A$.

Примечание. Начало делеции отмечено стрелкой.

\section{Заключение}

В обследованной иранской семье были диагностированы пациенты с $L Q-T S$ и СБ. Эти синдромы, как известно, опосредованы двумя различными механизмами нарушения функции натриевых каналов: увеличением и снижением входящего натриевого тока, соответственно. В данной семье в качестве генетической причины диагностированных синдромов была установлена делеция p.del KPQ1505-1507 в гене SCN5A, хотя эта мутация более известна как причина повышения функции натриевого канала и развития $L Q-T S$, но не СБ. Мы полагаем, что комбинированные признаки $L Q-T S$ и СБ у двоих членов семьи могут быть обусловлены дополнительной мутацией в других генах сердечных ионных каналов, потенциально вовлеченных в заболевание. Для проверки этой гипотезы необходим расширенный поиск мутаций в генах интереса. Такого рода исследование оптимально может быть выполнено с применением технологии высокоэффективного секвенирования нового поколения (next generation sequencing, NGS). Хотя пропранолол помогает контролировать клинические симптомы у членов этой семьи, для заключения об его эффективности необходимо длительное динамическое наблюдение, а носители мутаций должны быть информированы о максимально широкой комбинации факторов риска ВСС.

\section{REFERENCES}

1. Myerburg R.J., Kessler K.M., Castellanos A. Sudden cardiac death: epidemiology, transient risk, and intervention assessment. Ann. Intern. Med. 1993; 119 (12): 1187-1197.

2. Antzelevitch C., Brugada P., Brugada J., Brugada R., Shimizu W., Gussak I., Perez Riera A.R. Brugada syndrome: a decade of progress. Circ. Res. 2002; 91:1114-1118.

3. Wang Q., Shen J., Splawski I., Atkinson D., Li Z., Robinson J.L., Moss A.J., Towbin J.A., Keating M.T. SCN5A mutations associated with an inherited cardiac arrhythmia, long $Q-T$ syndrome. Cell. 1995; 80: 805-811.

4. McNair W.P., Ku L., Taylor M.R., Fain P.R., Dao D., Wolfel E., Mestroni L. Familial Cardiomyopathy Registry Research Group.
SCN5A mutation associated with dilated cardiomyopathy, conduction disorder, and arrhythmia. Circulation. 2004; 110: 2163.

5. Wang Q., Curran M.E, Splawski I., Burn T.C., Millholland J.M., VanRaay T.J., Shen J., Timothy K.W., Vincent G.M., de Jager T., Schwartz P.J., Towbin J.A., Moss A.J., Atkinson D.L., Landes G.M., Connors T.D., Keating M.T. Positional cloning of a novel potassium channel gene: $K V L Q-T 1$ mutations cause cardiac arrhythmias. Nat. Genet. 1996; 12: 17-23.

6. Curran M.E., Splawski I., Timothy K.W., Vincent G.M., Green E.D., Keating M.T. A molecular basis for cardiac arrhythmia: HERG mutations cause long $Q-T$ syndrome. Cell. 1995; 80: 795-803. 
7. Abriel H., Zaklyazminskaya E.V. Cardiacchannelopathies: genetic and molecular mechanisms. Gene. 2013; 517 (1): 1-11.

8. Indik J.H., Pearson E.C., Fried K., Woosley R.L. Bazett and Fridericia $Q-T$ correction formulas interfere with measurement of drug-induced changes in $Q-T$ interval. Heart Rhythm. 2006; 3 (9): 1003-1007.

9. Moric-Janiszewska E., Markiewicz Loskot G., Loskot M., Weglarz L., Hollek A., Szydlowski L. Challenges of diagnosis of long-Q $-T$ syndrome in children. Pacing Clin. Electrophysiol. 2007; 30 (9): 1168-1170.

10. Garg A., Lehmann M.H. Prolonged $Q-T$ interval diagnosis suppression by a widely used computerized ECG analysis system. Circ. Arrhythm. Electrophysiol. 2013; 6: 76-83.

11. Brugada P., Brugada J. Right bundle branch block, persistent $S T$ segment elevation and sudden cardiac death: a distinct clinical and electrocardiographic syndrome: a multicenter report. J. Am. Coll. Cardiol. 1992; 20: 1391-1396.

12. Priori S.G., Napolitano C., Gasparini M., Pappone C., Della Bella P., Giordano U., Bloise R., Giustetto C., De Nardis R., Grillo M., Ronchetti E., Faggiano G., Anni Nastoli J. Natural history of Brugada syndrome. Circulation. 2002; 105: 1342.

13. Duplyakov D.V., Glukhova V.L., Maksimova S.V., Vozhdaeva Z.I., Starostina I.V., Vasil'eva E.N., Sysuenkova E.V., Svetlakova L.P., Goleva S.V., Sorokina T.T. Kardiologiya - Cardology. 2007; 47(11): 55-59.

14. Zaklyazminskaya E.V., Abriel H. Prevalence of significant genetic variants in congenital long $Q-T$ syndrome is largely underestimated front. Pharmacol. 2012; 3: 72.

15. Nielsen M.W., Holst A.G., Olesen S.P., Olesen S.P. The genetic component of Brugada syndrome. Review article. Front. Physiol. 2013; 4: 179.

16. Moric E., Herbert E., Trusz-Gluza M., Filipecki A., Mazurek U., Wilczok T. The implications of genetic mutations in the sodium channel gene SCN5A. Europace. 2003; 5: 325-334

17. Wang Q., Shen J., Splawski I., Atkinson D., Li Z., Robinson J.L., Moss A.J., Towbin J.A., Keating M.T. SCN5a mutation associated with an inherited cardiac arrhythmia. Cell. 1995; 80: 805-811.

18. Benett P.B., Yazawa K., Makita N., George A.L., Jr. Molecular mechanism for an inherited cardiac arrhythmia. Nature. 1995; 376: 683-685.

19. Bezzina C., Rook M., Wilde A. Cardiac sodium channel and inherited arrhythmia syndromes. Cardiovasc. Res. 2001; 49: 257-271.

20. McNair W.P., Ku L., Taylor M.R., Fain P.R., Dao D., Wolfel E., Mestroni L.; Familial Cardiomyopathy Registry Research Group. SCN5A mutation associated with dilated cardiomyopathy, conduction disorder, and arrhythmia. Circulation. 2004; 110: 2163.

21. Deering T.F. Differential effects of beta blockers in patients with congenital long $Q-T$ syndromes. J. Am. Coll. Cardiol. 2012; 60: 2092-2099.

22. Priori S.G, Napolitano C., Schwartz P.J., Grillo M., Bloise R., Ronchetti E., Moncalvo C., Tulipani C., Veia A., Bottelli G., Nastoli J. Association of long $Q-T$ syndrome loci and cardiac events among patients treated with $\beta$-blockers. JAMA. 2004; 292 (11): 1341-1344.

23. Gollob M.H, Blier L., Brugada R., Champagne J., Chauhan V., Connors S., Gardner M., Green M.S., Gow R., Hamilton R., Harris L., Healey J.S., Hodgkinson K., Honeywell C., Kantoch M., Kirsh J., Krahn A., Mullen M., Parkash R., Redfearn D., Rutberg J., Sanatani S., Woo A. Recommendations for the use of genetic testing in the clinical evaluation of inherited cardiac arrhythmias associated with sudden cardiac death. Canadian J. Cardiol. 2011; 27: $232-245$.
24. Zareba W., Moss A.J., Daubert J.P., Hall W.J., Robinson J.L., Andrews M. Implantable cardioverter defibrillator in high-risk long $Q-T$ syndrome patients. J. Cardiovasc. Electrophysiol. 2003; 14 (4): 337-341.

25. Blaufox A.D., Tristani-Firouzi M., Seslar S., Sanatani S., Trivedi B., Fischbach P., Paul T., Young M.L., Tisma-Dupanovic S., Silva J., Cuneo B., Fournier A., Singh H., Tanel R.E., Etheridge S.P. Congenital long $Q-T 3$ in the pediatric population. Am. J. Cardiol. 2012; 109 (10): 1459-1465

26. Haissaguerre M., Derval N., Sacher F., Jesel L., Deisenhofer I., de Roy L., Pasquie J.L., Nogami A., Babuty D., Yli-Mayry S., De Chillou C., Scanu P., Mabo P., Matsuo S., Probst V., Le Scouarnec S., Defaye P., Schlaepfer J., Rostock T., Lacroix D., Lamaison D., Lavergne T., Aizawa Y., Englund A., Anselme F., O’Neill M., Hocini M., Lim K.T., Knecht S., Veenhuyzen G.D., Bordachar P., Chauvin M., Jais P., Coureau G., Chene G., Klein G.J., Clementy J. Sudden cardiac arrest associated with early repolarization. $N$. Engl. J. Med. 2008; 358 (19): 2016-2023.

27. Matsuo K., Yano K. Brugada syndrome. Nippon Rinsho. 2002; 60 (7): 1408-1414

28. Priori S.G., Aliot E., Lundqvist B., Bossaert C., Breithardt G., Brugada P., Camm A.J., Cappato R., Cobbe S.M., Di Mario C., Maron B.J., McKenna W.J., Pedersen A.K., Ravens U., Schwartz P.J., Trusz-Gluza M., Vardas P., Wellens H.J.J., Zipes D.P. Task force on sudden cardiac death of the European society of cardiology. Eur. Heart J. 2001; 22: 1374-1450.

29. Postema P.G., Mosterd A., Hofman N., Alders M., Wilde A.A. Sodium channelopathies: Do we really understand what's going on? J. Cardiovasc. Electrophysiol. 2011; 22 (5): 590-593.

30. Bezzina C., Veldkamp M.W., Van Den Berg M.P., Postma A.V., Rook M.B., Viersma J.W., van Langen I., Tan-Sindhunata G., Bink-Boelkens M.T., Van Der Hout A.H., Mannens M.M., Wilde A.A. A single $\mathrm{Na}(+)$ channel mutation causing both long- $Q-$ T and Brugada syndromes. Circ. Res. 1999; 85: 1206-1213.

31. Makita N., Behr E., Shimizu W., Horie M., Sunami A., Crotti L., Schulze-Bahr E., Fukuhara S., Mochizuki N., Makiyama T., Itoh H., Christiansen M., McKeown P., Miyamoto K., Kamakura S., Tsutsui H., Schwartz P.J., George A.L., Roden D.M. The E1784K mutation in SCN5A is associated with mixed clinical phenotype of type 3 long $Q-T$ syndrome. J. Clin. Invest. 2008; 118: 2219-2229.

32. Nuyens D., Stengl M., Dugarmaa S., Rossenbacker T., Compernolle V., Rudy Y., Smits J.F., Flameng W., Clancy C.E., Moons L., Vos M.A., Dewerchin M., Benndorf K., Collen D., Carmeliet E., Carmeliet P. Abrupt rate accelerations or premature beats cause life-threatening arrhythmias in mice with long $Q-T$ syndrome. Nat. Med. 2001; 7: 1021-1027.

33. Head C.E, Balasubramaniam R., Thomas G., Goddard C.A., Lei M., Colledge W.H., Grace A.A., Huang C.L. Paced electrogram fractionation analysis of arrhythmogenic tendency in DeltaKPQ Scn5a mice. J. Cardiovasc. Electrophysiol. 2005; 16: 1329-1340.

34. Tateyama M., Kurokawa J., Terrenoire C., Rivolta I., Kass R.S. Stimulation of protein kinase $\mathrm{C}$ inhibits bursting in disease-linked mutant human cardiac sodium channels. Circulation. 2003; 107: 3216-3222.

35. Abriel H. Role and regulation of the cardiac sodium channel NaV1.5: Recent insight from experimental studies. Cardiovasc. Res. 2007; 76: 381-389.

36. Cordeiro J.M., Barajas-Martinez H., Hong K., Burashnikov E., Pfeiffer R., Orsino A.M., Wu Y.S., Hu D., Brugada J., Brugada P., Antzelevitch C., Dumaine R., Brugada R. Compound heterozygous mutations $P 336 \mathrm{~L}$ and $11660 \mathrm{~V}$ in the human cardiac sodium channel associated with the Brugada syndrome. Circulation. 2006; 114: 2026-2033.

37. http://www.sads.org/living-with-sads/Drugs-to-Avoid/PrintableDrug-List

38. http://www.brugadadrugs.org/avoid/ 


\section{FOR CORRESPONDENCE}

Saber Siamak, consultant in genetics (cardiovascular genetics), I.M. Sechenov First Moscow State Medical University. Address: bld. 2, 8, Trubetskaya, Street, Moscow, RF, 119991; e-mail: s_saber2002@yahoo.com

Houshmand Massoud, Associate Professor at Department of Medical Biotechnology, National institute of Genetic Engineering and Biotechnology.

Address: Shahrak-e pajoohesh, km 15, Tehran-karaj Highway, Tehran, Iran, P.O.Box: 14965/161, Tehran, Iran, e-mail: housh62@yahoo.com

Samiei Nasab Mohammad Reza, Associate Professor at Chamran Heart Hospital, Isfahan University of Medical Sciences, Iran. Address: Bozorgmehr Bolivar, Salmane farsi Street, Chamran Heart Hospital, Isfahan, Iran,

e-mail: samieinasab@med.mui.ac.ir

Eftekharzadeh Mahmoud, Cardiologist, Electrophysiologist at Tehran Arrhythmia Center, Tehran, Iran.

Address: No: 30, Tavanir St.,Vali-Asr Ave.,Tehran, Iran, PO Box: 15175-536, e-mail: eftekhar@Iranep.org

Haghjoo Majid, Professor at Cardiac Electrophysiology Research Center, Rajaie Cardiovascular Medical and Research Center, Iran University of Medical Sciences, Tehran, Iran.

Address: Valie-Asr Street, Tehran,Iran, PO Box: 1996911151, e-mail: Majid.haghjoo@gmail.com

Fazelifar Amir-Farjam, Associate Professor at Cardiac Electrophysiology Research Center, Rajaie Cardiovascular Medical and Research Center, Iran University of Medical Sciences, Tehran, Iran.

Address: Valie-Asr Street, Tehran, Iran, PO Box: 1996911151, e-mail: fazelifar@gmail.com

Zaklyaz'minskaya Elena Valer'evna, PhD, professor of B.V. Petrovskii Russian Research Center for Surgery.

Address: 2, Abrikosovskii line, Moscow, RF, 119991; e-mail: helenezak@gmail.com

Gavrilenko Aleksandr Vasil'evich, PhD, academician of RAS, professor of B.V. Petrovskii Russian Research Center for Surgery.

Address: 2, Abrikosovskii line, Moscow, RF, 119991; e-mail: a.v.gavrilenko@mail.ru 